\title{
INNOVACIÓN DE LA EDUCACIÓN SUPERIOR EN EL ESTADO DE CHIHUAHUA
}

RICARDO ALMEIDA URANGA

\section{RESUMEN}

$O^{\prime}$ e presenta la implementación del proyecto de investigación titulado Sistematización de Experiencias Educativas en Comunidades de Práctica Innovadoras de la Red de Comunidades para la Renovación de la Enseñanza-Aprendizaje en Educación Superior de la Región Noroeste que se realizó en la clase de Filosofía de la Educación de la Licenciatura en Educación en la Universidad Autónoma de Ciudad Juárez durante dos ciclos escolares en 2018. Se logró modificar el modelo de enseñanza de la clase y se instrumentó en particular una secuencia didáctica para propiciar el desarrollo de las competencias de análisis, crítica y propuesta de una política pública del sector educativo en México a partir de diferentes perspectivas filosóficas.

Palabras clave: innovación e investigación educativa; transformación de la educación superior; problemas didácticos en educación superior; andamios pedagógicos; investigación acción participativa.

\section{ABSTRACT}

The implementation of the research project entitled "Systematization of Educational Experiences in Communities of Innovative Practice" is presented. This project was a research activity 
from the Network of Communities for the Renewal of TeachingLearning in Higher Education (Northwest Region in Mexico). The educational innovation was applied in the Philosophy of the Education class of the Bachelor of Education in the Autonomous University of Ciudad Juarez. The implementation was carried out during two school semesters in 2018. It was possible to modify the teaching model of the class and a didactic sequence was specially instrumented to promote the development of analytical and critical thinking applied to the understanding of public policy in the education sector in Mexico, standing from different philosophical perspectives.

\section{INTRODUCCIÓN}

La Subsecretaría de Educación Superior desarrolló a nivel nacional, durante el 2018, el primer año del proyecto de investigación educativa titulado la Sistematización de Experiencias Educativas en Comunidades de Práctica Innovadoras de la Red de Comunidades para la Renovación de la Enseñanza-Aprendizaje en Educación Superior (Proyecto ReCREA), el cual, siendo iniciativa de la Subsecretaría de Educación Superior, es coordinado por la Dirección General de Educación Superior para Profesionales de la Educación (DGESPE) y por la Dirección General de Educación Superior Universitaria (DGESU). Con el apoyo técnico de nueve escuelas normales y ocho universidades públicas estatales del país, se propuso que se realizara una investigación cuya preocupación temática inicial es la sistematización de experiencias educativas a través de la innovación de la práctica docente haciendo converger las siguientes tres perspectivas: (a) la visión del pensamiento complejo, (b) la investigación-acción en las aulas (c) y la integración de las tecnologías de la información y la comunicación (TIC) a la enseñanza.

Esta iniciativa reconoció la problemática que ha enfrentado la educación superior mexicana tanto en el subsistema de universidades públicas estatales como en el subsistema de las escuelas normales. En particular, el esfuerzo se dirigió a atender 
la recomendación que Cruz y Cruz (2008) propusieron para que la educación superior en México sea propiciatoria del desarrollo integral de los estudiantes. Cruz y Cruz (2008) recomiendan que en la educación superior mexicana es necesario "asegurar que los objetivos curriculares [de la educación superior en México] estén planteados en torno a la adquisición de competencias y habilidades, no sólo de conocimientos" (p. 15).

En el estado de Chihuahua, la Universidad Autónoma de Ciudad Juárez formó parte de los trabajos del Proyecto ReCREA con la participación de dos académicos.

En este reporte de investigación se presenta la documentación de la intervención de innovación educativa realizada en la clase de Filosofía de la Educación del programa de Licenciatura en Educación del Instituto de Ciencias Sociales y Administración de dicha universidad. También se exponen los resultados del primer año de investigación del Proyecto ReCREA, en particular para la clase de Filosofía de la Educación. La presente intervención, documentación y crítica fueron realizadas por el autor del presente trabajo.

\section{CONTEXTO DE LA INTERVENCIÓN}

La clase de Filosofía de la Educación (FE) fue utilizada para instrumentar y evaluar el modelo de innovación educativa propuesto desde el Proyecto ReCREA. Dicha clase se imparte en el programa de Licenciatura en Educación (LE) del Departamento de Humanidades del Instituto de Ciencias Sociales y Administración (ICSA) de la Universidad Autónoma de Ciudad Juárez (UACJ). El programa de LE consta de un total de 39 asignaturas obligatorias que cubren 312 créditos (8 créditos por clase). La anterior carga se completa con 80 créditos más que se consideran optativos. El número total de estudiantes de pregrado en la UACJ en el ciclo escolar 2016-2017 fue de 29 407; en el ICSA la población estudiantil ascendió en el ciclo escolar 2016-2017 a 7569. De dicha población, 968 estuvieron inscritos en el programa de LE, siendo 137 hombres y 831 mujeres (Duarte, 2017). La LE se encuentra acreditada 
hasta el 15 de enero de 2020 por el "Comité para la Evaluación de Programas de Pedagogía y Educación, A. C.” (CEPPE). En el portal del CEPPE (https://www.ceppe.org.mx/) se puede consultar la historia, los objetivos y el Marco de Referencia de dicho comité de evaluación. En el presente escrito se excluyen dichas precisiones, ya que excede los propósitos y el alcance de este trabajo.

El diseño curricular de la LE se divide en tres segmentos: Principiante, Intermedio y Avanzado. La clase de FE se ubica dentro del nivel intermedio. Citando la siguiente información del sitio en internet de la Universidad Autónoma de Ciudad Juárez (UACJ), se establece que el egresado del programa de la LE logrará el siguiente perfil:

(a) poseer la fundamentación teórico-metodológica sobre el origen y desarrollo de la pedagogía y de las ciencias de la educación, así como su ubicación en el campo humanístico y social; (b) asumir una postura crítica respecto al papel del profesional en educación y una actitud comprometida con la generación de estrategias acordes al contexto político, económico y social; (c) desarrollar actitudes inquisitivas y creativas ante la realidad que lo lleven a aplicar sus conocimientos, habilidades cognoscitivas y socio-profesionales para la evaluación y solución de los problemas que se plantean en la educación formal, no formal e informal; (d) poseer herramientas básicas en las diferentes áreas del quehacer educativo: tanto de docencia como de investigación y extensión, particularmente en las de educación superior, comunitaria, especial, gestión escolar y pedagogía industrial, que le permitan perfilar su futura orientación profesional (UACJ, 2019).

Asimismo, citando la misma fuente, se establece que el egresado de la LE potencialmente podrá abarcar los siguientes diez campos de trabajo:

- Docencia en los diferentes niveles educativos en instituciones públicas o privadas;

- Administración de las instituciones educativas; 
- Dirección de instituciones educativas;

- Coordinación de unidades académicas;

- Diseño de planes de estudio;

- Diseño y evaluación de programas de capacitación;

- Instructor de capacitación;

- Coordinación de programas educativos;

- Coordinación de programas comunitarios;

- Investigación educativa (UACJ, 2019).

En el ICSA se ofrecen clases tanto en el turno matutino -con horarios de 7:00 a 13:00 horas - como en el turno vespertino con horarios de 13:00 a 22:00 horas. La clase que se utilizó para la presente intervención educativa se impartió de 16:00 a 18:00 horas, es decir, en el turno vespertino. El grupo de FE estuvo constituido por quince estudiantes.

\section{SEMBLANZA SOCIODEMOGRÁFICA DEL GRUPO DE ESTUDIANTES}

La caracterización general del grupo se realizó describiendo las siguientes siete cualidades: género, edad, inserción laboral, localidad de nacimiento, estado civil, condición de paternidad/maternidad y las expectativas profesionales.

Género y edad. El grupo estuvo constituido por quince estudiantes, siendo cinco hombres (33.3\%) y diez mujeres (66.6\%). Las edades fluctuaron en un rango entre 19 y 28 años, siendo la edad promedio del grupo de 22 años. La desviación estándar de la edad fue igual a 3.39.

Inserción laboral. En el grupo once estudiantes se encontraban con trabajo formal, siendo el 73.3\% del total. La inserción laboral se dio de la siguiente manera: (a) dos estudiantes trabajaban en guarderías, (b) un estudiante trabajaba en una librería; (c) un estudiante trabajaba en una primaria bilingüe, (d) una de las estudiantes era guía en una escuela primaria Montessori, (e) un estudiante trabajaba en una papelería, (f) uno de los estudiantes trabajaba en una área de computación del Instituto de Ciencias Biomédicas de la UACJ, (g) dos estudiantes trabajaban en centros comerciales 
de cadenas norteamericanas instaladas en Ciudad Juárez, (h) un estudiante trabajaba en una maquiladora de la localidad, (i) un estudiante era emprendedor y administraba su propio negocio de lavado de carros.

Localidad de origen, estado civil y condición de paternidad/ maternidad. Doce estudiantes, es decir el 80\% del grupo, eran originarios de Ciudad Juárez; y tres estudiantes eran originarios de las ciudades de Torreón, Coahuila; Gómez Palacio, Durango; y una estudiante proveniente de la Ciudad de México. Del total, trece eran solteros, una estudiante divorciada y una más en unión libre. Únicamente una de las estudiantes era madre.

Expectativas profesionales. Las expectativas profesionales se listan a continuación. Los y las estudiantes expresaron de manera literal las siguientes aspiraciones personales:

- Incursionar en el ámbito de la capacitación en la industria fabril y maquiladora.

- Desempeñarse como docente en educación básica pública.

- Ser una persona emprendedora y fundar un jardín de niños(as).

- Incursionar en el análisis y generación de políticas públicas de educación eficaces.

- Ser una persona emprendedora y fundar una escuela Montessori.

- Dedicarse a la docencia en preescolar y/o ocuparse en la parte administrativa de escuelas de este nivel.

- Incursionar en la educación física en el nivel de educación básica.

- Desempeñarse como docente en educación superior.

- Continuar estudiando a nivel de maestría en la disciplina de sociología.

- Dedicarse a la enseñanza en las disciplinas de lectura y redacción.

- Insertarse en Educación Media Superior en la enseñanza de la disciplina de química. 
- Tener una plaza administrativa en la Secretaría de Educación Pública.

- Continuar estudios a nivel de maestría en psicología.

- Trascender a través de la enseñanza.

- Estudiar educación especial.

- Especializarse en estimulación temprana.

- Lograr la inserción laboral en El Paso, Texas, en Estados Unidos, en el sector educativo.

- No limitarse a obtener una plaza laboral en la burocracia gubernamental de la educación básica, es decir, dedicarse a un área educativa con mayor especialización.

- Dedicarse a la enseñanza en educación superior.

Para enmarcar brevemente el contexto del presente estudio se cita a continuación una muy sucinta semblanza de Ciudad Juárez:

Ciudad Juárez es una ciudad de México situada en el norte del país, en el estado de Chihuahua, a orillas del río Bravo. Al otro lado del río, en territorio estadounidense, se encuentra la ciudad de El Paso (Texas). Por su población de 1,428,508 habitantes según el informe de Plan Estratégico de Juárez 2018, es la mayor ciudad del estado de Chihuahua y la octava zona metropolitana más grande de México. Las dos ciudades fronterizas conforman la segunda zona metropolitana transnacional más grande de México y los Estados Unidos, que actualmente ronda los 2 millones aproximadamente de habitantes por lo cual, cuenta con una economía basada en la industria maquiladora formada por más de 200 empresas maquiladoras, las cuales están ubicadas estratégicamente en los puentes fronterizos y áreas de acceso rápido, cuentan con inversionistas estadounidenses, y su economía también es basada en la exportación de mercancía. La mayoría de los insumos son provenientes de Estados Unidos y las empresas son filiales estadounidenses que se instalan en México para aprovechar el bajo costo del factor mano de obra. Para no incurrir en costos de fletes y por cuestiones de logística gran parte de estas industrias están instaladas en ciudades a lo largo de la frontera: Matamoros, Reynosa y Nuevo Laredo al Este, Ciudad Juá- 
rez en el centro; y Nogales, Mexicali y Tijuana al oeste (Wikimedia Foundation, 2019).

\section{DISEÑO DE LA INTERVENCIÓN}

La presente intervención se generó a partir de la iniciativa de la Subsecretaría de Educación Superior (SES) que a través de la Dirección General de Educación Superior para Profesionales de la Educación (DGESPE) y de la Dirección General de Educación Superior Universitaria (DGESU) emitió la convocatoria para formar la Red de Comunidades para la Renovación de la EnseñanzaAprendizaje en Educación Superior (ReCREA) en el año 2017. En la conformación de dicha red se propusieron lineamientos metodológicos generales para orientar el proyecto titulado "Sistematización de Experiencias Educativas en Comunidades de Practica Innovadora".

Académicos de la Universidad Autónoma de Chihuahua (UACH), de la Universidad Autónoma de Ciudad Juárez (UACJ), de la Institución Benemérita y Centenaria Escuela Normal del Estado de Chihuahua "Profesor Luis Urías Belderráin" (IByCENECH), de la Escuela Normal Rural "Ricardo Flores Magón" (ENRRFM) y de la Benemérita y Centenaria Escuela Normal del Estado de Sonora "Profesor Jesús Manuel Bustamante Mungarro" (ByCENES) presentaron una contrapropuesta de instrumentación de los lineamientos generales de la DGESPE-DGESU (Subsecretaría de Educación Superior) del Proyecto RECREA para la Región Noroeste (RNO) de México. Dicha contrapropuesta condujo a que diversos investigadores de seis cuerpos académicos distintos de Chihuahua y Sonora integraran un grupo regional de trabajo para la renovación de la enseñanza-aprendizaje en educación superior. La conformación de dicha red llevó a implementar el Proyecto ReCREA de manera inicial en los estados de Chihuahua y Sonora. Posteriormente, en diciembre de 2018 se añadieron a la red de trabajo regional tres escuelas normales de los estados de Baja California (Escuela Normal Fronteriza Tijuana), Baja California Sur (Benemérita Escuela Normal 
Urbana Profr. Domingo Carballo Félix) y Sinaloa (Escuela Normal de Sinaloa).

Los investigadores que participaron en el Proyecto ReCREARNO provinieron de los cuerpos académicos e instituciones listados en la Tabla 1, haciendo la aclaración de que no necesariamente participaron, por diversas circunstancias, todos los miembros de los equipos que se listan. En el caso del CA-54-UACJ participaron dos investigadores: el Dr. Nolberto Acosta Varela y el Dr. Ricardo Almeida Uranga.

Tabla 1. Adscripción a grupos de investigación e instituciones de los académicos participantes en el Proyecto RECREA de Chihuahua y Sonora.

\section{Del estado de Chihuahua}

No. Adscripción de los investigadores Institución de Educación Superior participantes por grupo de inves-

tigación

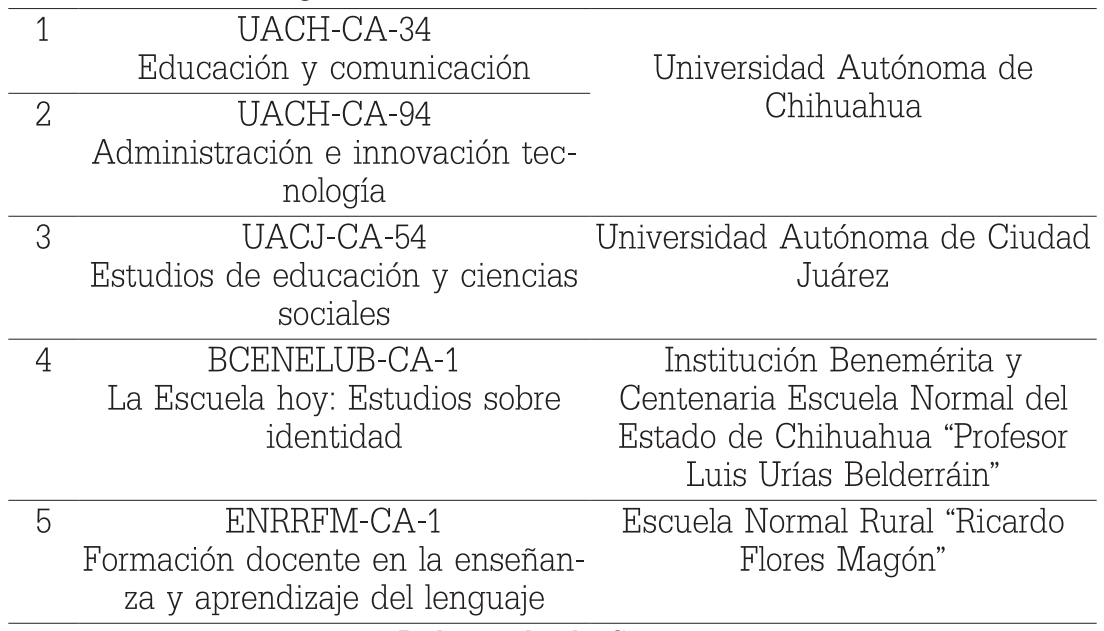

Del estado de Sonora

No. Adscripción de los investigadores Institución de Educación Superior participantes por grupo de investigación

$6 \quad$ ENEJMBM-CA-1

Docencia, prácticas educativas y

su impacto social

Benemérita y Centenaria Escuela Normal del Estado de Sonora "Profesor Jesús Manuel Bustamante Mungarro"

Fuente: elaboración propia. 


\section{FUNDAMENTACIÓN CONCEPTUAL DEL PROYECTO RECREA-RNO}

Se presenta en la Figura 1 el modelo teórico-metodológico desarrollado por la DGESPE-DGESU (SES-SEP) en el que se basó de manera central la propuesta de intervención para orientar la mejora de la enseñanza-aprendizaje en educación superior en las universidades y escuelas normales de la Región Noroeste de México.

En dicho esquema se identifican las tres perspectivas (pensamiento complejo, investigación-docencia [investigación-acción] y uso de las TIC) que se mencionaron previamente para guiar el trabajo investigativo en las asignaturas elegidas por los docentes de cada institución.

Figura 1. Enfoque teórico-metodológico del Proyecto ReCREA-RNO.

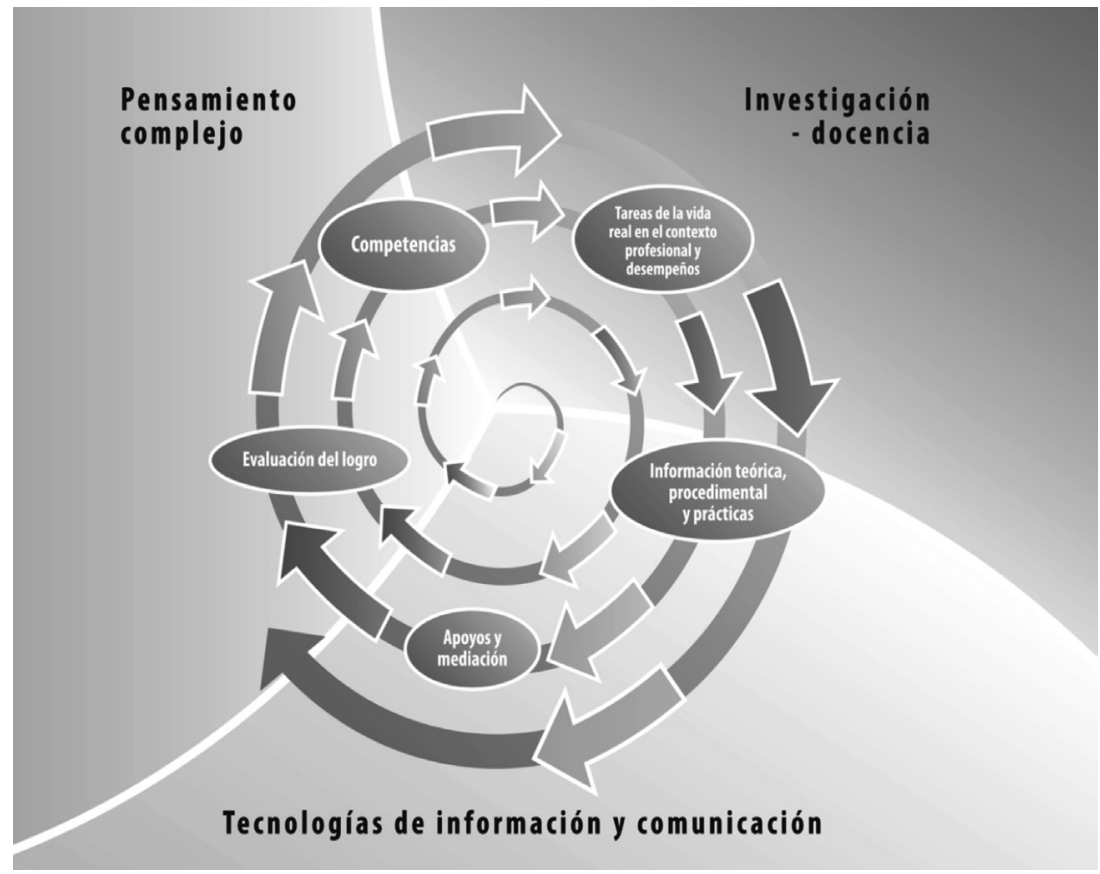

Fuente: Dirección General de Educación Superior, ReCREA en Educación Superior (Subsecretaría de Educación Superior, Secretaría de Educación Pública, 2018). 
Dichos referentes teórico-metodológicos fueron utilizados como directrices generales del presente esfuerzo de innovación educativa en educación superior inicialmente en la clase de Filosofía de la Educación.

Se presenta a continuación una interpretación propia de los conceptos básicos del Pensamiento Complejo del modelo de intervención propuesto por el Proyecto ReCREA que fueron utilizados de manera parcial para dirigir la intervención de innovación educativa en la asignatura señalada del programa de la Licenciatura en Educación (ICSA-UACJ). También se explica su instrumentación en la elaboración de herramientas pedagógicas que permitieron aplicar de manera concreta la innovación.

El pensamiento complejo. Se inicia la descripción de esta arista citando a Pakman (1994) en la introducción que escribió de la colección de varios escritos realizados por Morin entre 1976 y 1988 y publicados bajo el título "Introducción al pensamiento complejo". Pakman (Prólogo escrito en Morin, 1994) dice:

Cuando nos asomamos a entender al mundo físico, biológico, cultural en el que nos encontramos, es a nosotros mismos a quienes descubrimos y es con nosotros mismos con quienes contamos. El mundo se moverá en una dirección ética, sólo si queremos ir en esa dirección. Es nuestra responsabilidad y nuestro destino el que está en juego. El pensamiento complejo es una aventura, pero también un desafío (p. 9).

La idea inicial expresada por Pakman ubica en el centro del cambio a la persona en sí misma. El pensamiento complejo tal como lo desarrolla Morin es una reflexión sobre el desarrollo de la ciencia positivista y los resultados de su aplicación en las sociedades contemporáneas. Uno de los primeros puntos en señalar desde esta perspectiva es que "[e]n este sentido el pensamiento complejo aspira al conocimiento multidimensional. Pero sabe, desde el comienzo, que el conocimiento completo es imposible" (Morin, 1994, p. 11). Morin intenta llamar la atención sobre la fragmentación del conocimiento y propone una reflexión en torno a los re- 
duccionismos de las disciplinas académicas. Morin (1994) propone que es así que "el pensamiento complejo está animado por una tensión permanente entre la aspiración a un saber no parcelado, no dividido, no reduccionista, y el reconocimiento de lo inacabado e incompleto de todo conocimiento" (p. 11). De aquí se puede apreciar una contradicción inherente en la perspectiva moriniana: por un lado, propone la visión integrada y completa de la realidad, y simultáneamente reconoce que lo anterior no es posible:

La patología de la razón es la racionalización, que encierra a lo real en un sistema de ideas coherente, pero parcial y unilateral, y que no sabe que una parte de lo real es irracionalizable, ni que la racionalidad tiene por misión dialogar con lo irracionalizable (Morin, 1994, p. 19).

Morin reconoce que el alcance de la mente analítica-racional es limitado y no puede abarcar la completitud; asimismo, declara que dicho tope establece que lo que está fuera del alcance de la racionalidad humana tampoco puede ser sujeto de diálogo racional: "[e]sta restricción de conceptos, esta fisura ontológica, esta regresión de la objetividad, del determinismo, parecen aportar, como primer fruto, la regresión general del conocimiento, la incertidumbre ... " (Morin, 1994, p. 42). Dicho reconocimiento de las limitaciones de la mente racional-analítica le permiten a Morin reconocer la condición humana de la incertidumbre. La propuesta de Morin es una invitación a la reflexión sobre las disciplinas académicas de las instituciones de educación superior. Lo plantea de la siguiente manera: "[v]amos a intentar aquí un discurso multidimensional no totalitario, teórico, pero no doctrinario ... abierto a la incertidumbre y a la transcendencia; no ideal/idealista, sabiendo que la cosa no será nunca totalmente encerrada en el concepto, el mundo jamás aprisionado en el discurso" (Morin, 1994, p. 46-7).

De la incongruencia que plantea entre la visión del todo versus su propia imposibilidad, intenta resolver la contradicción planteando que: "[s]i, efectivamente, el espíritu humano no puede aprehender el enorme conjunto del saber disciplinario, hace falta, entonces, cambiar, ya sea al espíritu humano, ya sea al saber 
disciplinarizado" (Morin, 1994, p. 49). Dicha disyuntiva es tema de reflexión para las distintas audiencias receptoras del mensaje de Morin. En la presente intervención se trabajó tanto con las personas, es decir, el "espíritu humano", hablando metafóricamente en términos de Morin, y también se le imprimió una perspectiva innovadora a la forma de presentar el saber organizado en disciplinas para intentar un cierto grado de congruencia epistemológica con las tesis del pensamiento complejo moriniano.

Morin intenta asociar las situaciones caóticas que se han generado en la sociedad humana contemporánea con los enfoques y paradigmas del propio pensamiento humano: "[p]orque es, evidentemente, toda la estructura del sistema de pensamiento la que se halla trastornada, es toda una enorme estructura de ideas la que colapsa. He aquí aquello para lo cual hay que prepararse" (Morin, 1994, p. 52). Desde esta tesitura intrínsecamente contradictoria, Morin también aborda la paradoja de la libertad de la expresión humana versus los múltiples determinismos a los que las personas están sujetas:

Cuán a menudo tenemos la impresión de ser libres sin ser libres. Pero, al mismo tiempo, somos capaces de libertad, del mismo modo que somos capaces de examinar hipótesis de conducta, de hacer elecciones, de tomar decisiones. Somos una mezcla de autonomía, de libertad, de heteronomía e incluso, yo diría, de posesión de fuerzas ocultas que no son simplemente las del inconsciente, descubiertas por el psicoanalista. He aquí una de las complejidades propiamente humanas (Morin, 1994, p. 62).

Las anteriores reflexiones se presentan a manera de introducción al pensamiento complejo y a los retos que plantea. No es la intención del actual trabajo la de presentar tesis que respondan, o intenten resolver, los dilemas filosóficos planteados por Morin, sino ponerlos de relieve.

Para el propósito del Proyecto ReCREA-RNO, que fue el de innovar la educación superior en comunidades de práctica siguiendo líneas generales de la investigación social-colectiva y utilizan- 
do las TIC, el marco de referencia provisto por Morin proporcionó un marco de reflexión filosófico que permitió fundamentar dos propósitos: (a) el primero de ellos es provocar la reflexión crítica de los alcances del quehacer docente e investigativo en las aulas de las universidades y de las escuelas normales, en este caso, de los estados de Chihuahua y Sonora, y del resto de la región noroeste (NO) del país; (b) el segundo, las ideas del pensamiento complejo pudieron ser utilizadas para orientar la planeación didáctica del profesor universitario y normalista que a su vez dieron pie a la instrumentalización del enfoque a través del diseño de andamios de aprendizaje. Dichos andamios pedagógicos se realizaron para guiar el trabajo de enseñanza por parte de los docentes y el aprendizaje por parte de los estudiantes de educación superior.

\section{MÉTODO DEL PROYECTO RECREA-RNO}

El Proyecto RrCREA a nivel nacional ofreció directrices generales en relación al método proporcionando el siguiente conjunto básico de referentes: Aseguramiento de la Calidad en la Educación y en el Trabajo, S. C. (2010b); Bausela (2004); Gulikers, Bastiaens y Kirschner (2004); Jenkins et al. (2007); Kirschner, Sweller y Clark (2006); Morin (2002); Nicolescu (1996, 2006a, 2006b); Norton (2009); Osorio (2012); Padilla y Gil (2008); Roegiers (2010); Sluijsmans, Straetmans y Van Merriënboer (2008); The Boyer Commission for Educating Undergraduates in the Research University (2001); y Van Merriënboer y Kirschner (2010).

El grupo de trabajo del Proyecto ReCREA-RNO, al revisar la propuesta y el conjunto de referentes teóricos (algunos de ellos citados en el párrafo anterior) para el trabajo académico de la Red CREA por parte de la DGESPE-DGESU observamos una convergencia teórico-metodológica con el Modelo para el Desarrollo y Evaluación de Competencias Académicas (M-DECA) desarrollado con anterioridad por académicos pertenecientes al Cuerpo Académico no. 34 "Educación y Comunicación" de la Universidad Autónoma de Chihuahua (Cisneros-Cohemour, García-Cabrero, Luna y Marín, 2012; Guzmán y Marín, 2015; Guzmán, Marín e Inciarte, 
2014; Marín y Guzmán, 2012). Al formar parte dicho cuerpo académico del equipo de trabajo para la región noroeste de la Red CREA, ello coadyuvó a interpretar e instrumentar las directrices nacionales para la innovación educativa de manera más operativa. Lo anterior agilizó la implementación del proceso de investigación en la región $\mathrm{NO}$.

Instrumentación de la innovación mediante andamios pedagógicos. Tomando en cuenta la convergencia tanto teórica como de método entre los referentes del Proyecto ReCREA a nivel nacional y el M-DECA se decidió utilizar las herramientas de los andamios didácticos para implementar y evaluar la innovación en educación superior. El andamiaje pedagógico desarrollado permitió instrumentar las directrices pedagógicas propuestas por la DGESPE-DGESU. En el caso de la clase de Filosofía de la Educación la intervención fue concretada mediante la elaboración de dos andamios diseñados para este propósito por el grupo de la red de la región NO basándose en las directrices proporcionadas por Guzmán y Marín (2015), Marín y Guzmán (2012) y por Guzmán, Marín e Inciarte (2014). La doctora Isabel Guzmán Ibarra y el doctor Rigoberto Marín Uribe (adscritos a la UACH) también formaron parte del grupo de investigación del Proyecto ReCREA-RNO, por lo que en todo momento se contó con su asistencia pedagógica.

Andamiaje pedagógico. El primer andamio fue de planeación didáctica para orientar al profesor sobre la conducción de la innovación educativa en clase. El segundo andamio se elaboró a partir del anterior y estuvo dirigido al grupo de estudiantes como herramienta para el desarrollo de competencias.

Para la planeación didáctica se tomaron en cuenta en la elaboración de los andamios las siguientes tres ideas (llamadas principios por Morin, 1994) del pensamiento complejo:

La perspectiva dialógica. En la innovación educativa se estimuló el diálogo entre todos los participantes de la clase Filosofía de la Educación, así como la revisión de posturas filosóficas distintas. También se propició el diálogo metafórico entre posturas del pensa- 
miento filosófico que pueden interpretarse como antagónicas, diferentes o contrastantes.

La recursividad organizacional. Se propuso una forma de razonamiento de espiral y no lineal dando a entender que una misma condición puede ser simultáneamente efecto y causa de la situación que lo provocó.

La dimensión hologramática. En relación con esta idea se analizó, cuando fue pertinente, las condiciones de la realidad en donde la estructura mayor es contenida simultáneamente por una de sus partes como las relaciones y los efectos que se dan en políticas públicas del sector educativo de los tres niveles de gobierno en México: federal, estatal y municipal.

Se utilizaron durante dos periodos académicos semestrales consecutivos (enero-mayo y agosto-diciembre 2018) los dos andamios, el que sirvió de guía para el profesor titular de la clase, y el andamio del estudiante. Se trabajó en esta intervención con una sola secuencia didáctica en la clase que fue considerada como transversal, es decir, fue recurrentemente vista y revisada a lo largo de todo el periodo académico. La secuencia didáctica elegida para esta clase fue el análisis crítico desde una o varias ópticas filosóficas, de una política pública del sector educativo en México elegida para dicho fin. El estudiante fue responsable de seleccionar la política pública a analizar y criticar; y también fue responsable de decidir la postura o las posturas filosóficas desde las cuales tendría que realizar el análisis crítico de la política pública del sector educativo en México.

La planeación didáctica en el andamio del docente, se realizó en seis fases (pasos) que correspondieron a los segmentos de trabajo propuestos por la DGESPE-DGESU (SES-SEP) y fundamentados en referentes proporcionados por el Aseguramiento de la Calidad en la Educación y en el Trabajo, S. C. (ACET, 2010a, 2010b, 2010c) para instrumentar la innovación educativa. Dichas fases (llamados pasos en los documentos de trabajo) fueron los siguientes: (a) contexto de la asignatura; (b) tareas y proyectos de aprendizaje complejo; (c) información teórica, procedimental y 
prácticas; (d) apoyos y mediación; (e) evaluación del desempeño; (f) presentación a los estudiantes.

La estructura del andamio pedagógico que se diseñó para el grupo de estudiantes se presenta en la Tabla 2.

Tabla 2. Secciones del andamio del estudiante.

\begin{tabular}{|c|c|}
\hline$\overline{\mathrm{Ob}}$ & de estudio \\
\hline & entación \\
\hline & Descripción \\
\hline & Intenciones formativas \\
\hline & Competencias de salida y constitutivas \\
\hline II. & Dispositivo de formación \\
\hline & Situación problema \\
\hline & Actividades de aprendizaje \\
\hline & Evidencias de desempeño \\
\hline & Recursos de apoyo \\
\hline & Dispositivo de evaluación \\
\hline & Referencias bibliográficas \\
\hline
\end{tabular}

En la parte II del andamio del estudiante, titulada Dispositivo de Formación, refiriéndose a la situación-problema, es en donde se aplicaron los tres principios propuestos por Morin (1994) referentes a lo dialógico, a lo recursivo y a lo hologramático. Las preguntas guía que se utilizaron para la reflexión con el grupo de estudiantes se presentan en la Tabla 3.

Tabla 3. Dispositivo de formación referente a la Situación-problema

1. Dialógico: la unidad de antagonismos

a. ¿Qué argumentos son los más críticos de la Política Pública de Educación (PPE)?

b. ¿Qué contradicciones expresa la PPE?

C. ¿Cómo se pueden resolver las contradicciones de la PPE?

2. Recursión: ¿qué consecuencias se pueden generar con diferentes alternativas de Política Pública de Educación?

a. ¿Hay otras opciones a la PPE planteada? 
b. Las situaciones que genera la PPE, ¿actúan como causas de las condiciones que pretenden resolver?

c. Cuestionamiento crítico de la eficacia de la PPE.

3. Hologramático. En los sistemas "familia", "aula”, "escuela", "municipio", "entidad", "país":

a. ¿Cómo se manifiesta la situación a la que hace referencia la PPE?

b. ¿Cómo se reproducen las condiciones sociales y educativas?

c. Identificación de espirales "virtuosas" y "viciosas".

d. ¿Cómo se pudiesen detonar espirales "virtuosas"?

e. ¿Cómo se pudiesen transformar espirales "viciosas" en espirales "virtuosas"?

Para complementar la formulación anterior, la clase de Filosofía de la Educación se diseñó teniendo como orientación pedagógica las siguientes carácterísticas: (a) la de ser una clase activa, (b) autodirigida, (c) vivencial, (d) propiciatoria de un ambiente de cooperación, (e) y conformando una comunidad de aprendizaje.

El producto final que se propuso para el desarrollo de competencias en un ambiente de complejidad fue un ensayo. La estructura de dicho ensayo final se presenta en la Tabla 4.

Tabla 4. Secciones del ensayo de análisis de política pública en educación.

a. Introducción. Una breve descripción del tipo de trabajo que se presenta redactando una caracterización general de la clase Filosofía de la Educación.

b. Descripción de la política pública elegida. Especificación de la Política Pública en Educación elegida, explicando por qué se eligió esa política en particular. Presentación de una descripción general de la política y una descripción de la línea de política pública específica cuyo enfoque se aborda para su crítica.

c. Perspectiva(s) filosófica(s) aplicada(s) en el análisis crítico. Explicación sintética del(os) enfoque(s) filosófico(s) desarrollado(s) en el reporte académico, utilizando mapas conceptuales. 
d. Análisis crítico de la política pública. Explicación de los diferentes puntos de la política pública que son criticados desde una o varias perspectivas filosóficas y fundamentar las críticas de manera amplia. Aplicación de los principios dialógico, recursivo y hologramático para su análisis.

e. Recomendaciones para mejorar la política pública expuesta. Derivado de la crítica, llevar a cabo la realización de propuestas de rediseño o de reorientación de la política pública para hacerla más congruente, más eficaz y pertinente.

f. Conclusiones generales. Aprendizajes principales derivados del análisis crítico y de la clase de Filosofía de la Educación.

g. Referencias bibliográficas. Utilización del Manual de Presentación de Trabajos y Exámenes (Almeida, Arias, Curiel, Díaz, Flores t, Muttio y Priego, 2009).

h. Anexos de interés. Si los hubiere.

Los criterios que se tomaron en cuenta para determinar el grado de desarrollo de la competencia del grupo de estudiantes para el análisis de políticas públicas en educación en México se presentan en la Tabla 5.

Tabla 5. Criterios de revisión y retroalimentación de la competencia constitutiva.

\begin{tabular}{c}
\hline Uso de conceptos vistos en clase. \\
\hline Nivel de la argumentación \\
\hline Consistencia y articulación lógica. \\
Claridad \\
\hline Originalidad en las aportaciones. \\
\hline Uso de fuentes de información adicionales a las asignadas para la clase. \\
\hline Uso de citas y referencias bibliográficas. \\
Relación entre diversos temas. \\
\hline Respeto al formato y consistencia del mismo.
\end{tabular}

La intención formativa de la clase de FE se presenta en la Tabla 6. 
Tabla 6. Intención formativa en la clase de Filosofía de la Educación.

A partir del conocimiento de diversos modelos conceptuales de análisis
filosófico, desarrollar competencias para aplicar algunos elementos de
perspectivas filosóficas elegidas por el(la) estudiante, con objeto de abor-
dar críticamente propuestas de política pública en el campo de la edu-
cación obligatoria mexicana, dentro de escuelas del nivel básico y medio
superior.
Basado en dicha aplicación crítica de enfoques filosóficos elegidos por
el(la) estudiante, proponer estrategias de intervención educativa alterna-
tivas y/o recomendaciones de orientación de política pública que sean
pertinentes a la población estudiantil mexicana, particularmente en ciu-
dad Juárez y en el estado de Chihuahua, expresando una fundamenta-
ción conceptual, documental, argumentativa y lógica.

Dicha expresión y proposición crítica se realizará dentro del contexto de la sociedad compleja y utilizando las tecnologías de la información y de la comunicación.

Las competencias constitutivas planteadas se presentan en la Tabla 7.

Tabla 7. Competencias constitutivas de la clase Filosofía de la Educación.

\section{Competencia constitutiva 1}

$\overline{\text { El estudiante identifica, elige y sintetiza una o dos perspectivas filosóficas }}$ contemporáneas que tienen aplicabilidad en el sector educativo. En dicha síntesis, el estudiante destaca sus implicaciones de manera inicial para la educación mexicana, particularmente en Ciudad Juárez y en el estado de Chihuahua.

Competencia constitutiva 2

El estudiante elige de la propuesta oficial de la SEP titulada "Modelo Educativo para la educación obligatoria, educar para la libertad y la creatividad" (SEP, 2017), para su análisis, una de las vertientes de política pública de alguna de sus secciones: (I) el planteamiento curricular; (II) la escuela al centro del sistema educativo; (III) formación y desarrollo profesional de los maestros; (IV) inclusión y equidad; (V) y la gobernanza del sistema educativo. El estudiante puede utilizar otros documentos de PPE de la SEP para su elección.

Competencia constitutiva 3 
Basándose en algunos elementos conceptuales de la(s) perspectiva(s) filosófica(s) elegida(s), el(la) estudiante propone estrategias de intervención educativa alternativas y/o recomendaciones de orientación de política pública que sean pertinentes a la población estudiantil mexicana, particularmente en Ciudad Juárez y en el estado de Chihuahua, expresando una fundamentación conceptual, documental, argumentativa y lógica.

La descripción realizada hasta este punto delinea solo una arista del modelo de intervención realizada en la clase de Filosofía de la Educación del programa de Licenciatura en Educación del Departamento de Humanidades del ICSA-UACJ. Dicha vertiente fue la del Pensamiento Complejo. Se explicó tanto una interpretación propia de su propuesta teórica general como su instrumentación en clase.

En el siguiente apartado se explican los otros dos vértices del modelo de innovación educativa del Proyecto ReCREA: (a) el de investigación y docencia, (b) y el de uso y aplicación de las TIC.

Dichos referentes cumplieron simultáneamente con dos fases del proceso de investigación educativa que se implementó: (a) la intervención, (b) y el de evaluación de la innovación educativa en el aula. Ambos temas se explican a continuación.

\section{SEGUIMIENTO Y EVALUACIÓN: LA INVESTIGACIÓN-ACCIÓN Y EL USO DE LAS TIC}

El estudio se llevó a cabo en los semestres de primavera (eneromayo) y otoño (agosto-diciembre) de 2018. La formación profesional en las universidades públicas estatales (UPE) y en las escuelas normales de México del siglo XXI presenta una problemática caracterizada por el crecimiento exponencial de la complejidad y por la incertidumbre que se deriva de dicha condición. Los profesionistas requieren de una actualización permanente en cuanto a los conocimientos relacionados con sus respectivas disciplinas. Esta problemática se ve acentuada por el desarrollo de las tecnologías de la información y de la comunicación (TIC) en un mundo globalizado. El dinamismo y la complejidad del entorno influyen para que los problemas de las distintas profesiones cada vez presen- 
ten mayor ambigüedad y mayores dificultades para definirlos con precisión. Para enfrentar dichos retos de la sociedad contemporánea, por parte del Proyecto ReCREA (DGESPE-DGESU, SES-SEP) se diseñó un modelo de innovación educativa orientado hacia el desarrollo de competencias que puede ser aplicado por distintas profesiones y áreas disciplinarias, tanto en las UPE como en las escuelas normales.

La metodología de indagación, cuestionamiento y deliberación aplicada en el presente estudio es la de Investigación-Acción Participativa (IAP). Se consultaron diversas fuentes tales como Elliot (1993); Giroux y Tremblay (2004); Jenkins, Healey y Zetter (2007); Kemmis (1998); Kemmis y MacTaggart (1992, 2007); Latorre (2005); Norton (2009); Rodríguez y Valldeoriola (2009) y Shu (2016) para diseñar e intervenir en el aula en programas de pregrado directamente. Se ha enfatizado el modelo desarrollado por la Universidad de Deakin:

La investigación-acción es una forma de indagación introspectiva colectiva emprendida por participantes en situaciones sociales con objeto de mejorar la racionalidad y la justicia de sus prácticas sociales o educativas, así como su comprensión de esas prácticas y de las situaciones en que éstas tienen lugar (Kemmis y McTaggart, 1992, p. 9).

La investigación-acción es eminentemente colaborativa y crítica de las propias acciones de las personas que constituyen el o los equipos de investigación. La investigación consiste en un número indefinido de ciclos de investigación en los que se siguen cuatro fases que se detallan en la Tabla 8.

Tabla 8. Fases de la investigación-acción participativa (Kemmis y McTaggart, 1992, 2007)

I. La planeación de una acción o de varias acciones concretas.

II. Implementación de la acción o de la secuencia de acciones acordada.

III. Observación sistemática de la conducción de las acciones y de sus diversos impactos. 
IV. Reflexión y socialización crítica, con base en lo observado, con la finalidad de planear una acción o secuencia de acciones nuevas.

Con el paso cuatro se inicia de nuevo el ciclo de investigación. La unidad de análisis u objeto de estudio del Proyecto ReCREARNO (DGESPE-DGESU, SES-SEP) fue la propia práctica educativa de los integrantes del equipo de investigadores a nivel regional.

Para la región noroeste del Proyecto ReCREA se invitó a colaborar a investigadores interesados y con disponibilidad para participar adscritos a seis equipos de investigación constituidos previamente como cuerpos académicos (ver Tabla 1). Participaron académicos de las siguientes instituciones: (a) Universidad Autónoma de Chihuahua (dos cuerpos académicos), (b) Universidad Autónoma de Ciudad Juárez, (c) Institución Benemérita y Centenaria Escuela Normal del Estado de Chihuahua "Profesor. Luis Urías Belderráin", (d) Escuela Normal Rural de Saucillo "Ricardo Flores Magón”, (e) y de la Benemérita y Centenaria Escuela Normal del Estado de Sonora "Profesor Jesús Manuel Bustamante Mungarro".

Se constituyó un séptimo colectivo con los(as) profesores(as) de las instituciones anteriores que tuvieron tanto el interés como la disponibilidad para participar en el Proyecto ReCREA-RNO. Dicha red de académicos se constituyó como grupo plenario de trabajo para la investigación-acción regional para los estados de Chihuahua y Sonora. En diciembre de 2018 se amplió el grupo para incluir académicos de los estados de Baja California, Baja California Sur y Sinaloa.

El grupo de académicos participantes inició en la primavera del 2018 (semestre enero-mayo) una exploración inicial (estudio piloto) con los fundamentos del Proyecto ReCREA y durante el periodo académico semestral de otoño (agosto-diciembre) del mismo año se estuvo implementando de manera íntegra a lo largo de todo el periodo académico la innovación educativa empleando los andamios pedagógicos. La investigación se inició en marzo de 2018 con el reconocimiento de la necesidad del cambio y se realizó la planeación inicial. Durante el primer semestre y el verano de 2018 
se hizo la fase de planeación, diseñando y utilizando los andamios para el profesor y para los estudiantes. El modelo del proyecto ReCREA se documentó y sistematizó durante el semestre de otoño de 2018. En el caso de la presente documentación, la innovación educativa se aplicó al grupo de Filosofía de la Educación con un total de quince estudiantes. Esta clase se ofrece en el nivel intermedio del plan de estudios de la Licenciatura en Educación del Departamento de Humanidades (ICSA-UACJ).

Los dos integrantes del equipo de investigación de la UACJ nos reunimos una vez cada quince días, en sesiones con una duración promedio de dos horas, durante ambos semestres, para un total de 16 sesiones. Para la clase de Filosofía de la Educación se invitó a participar como miembros del equipo de reflexión y análisis a los quince estudiantes de dicha clase del semestre de otoño (agosto-diciembre) de 2018. Con ellos se tuvieron sesiones de intervención-acción semanales durante los periodos de la clase. En total se completaron 10 sesiones de valoración crítica del material utilizado para la clase. Con el equipo de investigación regional se llevaron a cabo durante el año 2018 un total de seis sesiones con las personas investigadoras de las dos UPE del estado de Chihuahua (UACJ y UACH), de las dos escuelas normales del estado de Chihuahua (IBYCENECH, ENRRFM) y de la escuela normal del estado de Sonora (BYCENES). Finalmente, el grupo de la región noroeste del Proyecto ReCREA realizó dos ciclos amplios de investigación, uno para el semestre enero-mayo y el segundo para el semestre agosto-diciembre de 2018, con sesiones cortas de reflexión y análisis dentro de los mismos periodos semestrales, y un intervalo intersemestral correspondiente al verano de 2018.

Durante los anteriores ciclos (desagregados a nivel de profesor, por institución participante y regional), se llevó a cabo una valoración académica del modelo del Proyecto ReCREA (presentado en la Figura 1).

En la clase de Filosofía de la Educación, el andamiaje proporcionado al grupo de estudiantes estableció las condiciones específicas que debían generarse en el ambiente áulico para orientar los contenidos y el proyecto académico transversal hacia su aplica- 
ción práctica en la profesión de Licenciado en Educación. Dichas condiciones se presentan en la Tabla 9.

Tabla 9. Condiciones de aplicación del contenido y del proyecto académico transversal.

a. La presentación de políticas públicas del sector educativo intrínsecamente complejas en un contexto también de ambigüedad y dinamismo por la transición gubernamental que se vivió en México y particularmente en Ciudad Juárez en los tres niveles de gobierno: municipal, estatal y federal.

b. Énfasis en el carácter incierto de las condiciones de desempeño debido al movimiento e incertidumbre en relación a la conducción futura que se estará teniendo en el siguiente año para el diseño, implementación y evaluación de las políticas públicas en educación.

c. Se plantearon requerimientos y necesidades de evaluación orientados al desarrollo progresivo de competencias de análisis de políticas públicas en el sector educativo.

d. Incluir el uso y aplicación de las TIC para la investigación de las problemáticas educativas de acuerdo con los siguientes recursos: utilización de bases de datos, fuentes de información documental, sitios de investigación académica, uso de los portales de información gubernamentales y en la preparación de exposiciones y temáticas relacionadas con los temas de la clase de FE.

La colegiación del análisis, de la reflexión y de la crítica se logró en los tres niveles de integración de los equipos de investigación: a nivel áulico con el grupo de estudiantes de FE; a nivel institucional con dos profesores pertenecientes al Cuerpo Académico 54 Estudios de Educación y Ciencias Sociales de la UACJ; y a nivel regional en la interacción entre los investigadores de las cinco instituciones de educación superior.

\section{TÉCNICAS UTILIZADAS PARA DOCUMENTAR EL CAMBIO}

Andamiaje pedagógico y observación participante. Rekalde, Vizcarra y Macazaga (2014) emplearon la metodología de investiga- 
ción-acción a través de la técnica de observación participante. Ellas plantean que: "[e]sta apuesta ha significado la implicación de un grupo de personas que han optado conscientemente por un cambio socio-educativo ...." (p. 202). Lo anterior ocurrió con la intervención del Proyecto ReCREA en los tres niveles:

- Áulico: profesor con el grupo de estudiantes de la clase de Filosofía de la Educación.

- Dos investigadores de la UACJ adscritos nominalmente al CA-54 Estudios de Educación y Ciencias Sociales.

- Colectivo regional para la planeación e innovación educativa de Chihuahua y Sonora.

En el caso de la clase de FE se propició una comunicación horizontal con el grupo de estudiantes que favoreció el diálogo. La estrategia central la constituyó la observación participante por parte del profesor titular de la clase, quien también fue líder del cuerpo académico. Los objetivos de la investigación-acción participativa fueron: (a) reflexionar críticamente sobre las actividades académicas diseñadas desde el Proyecto ReCREA-RNO, (b) aplicar y modificar con base en la experiencia los andamios pedagógicos que se desarrollaron como medio de instrumentación de la innovación didáctica.

Bolívar, De la Cruz y Domingo (2001) explican que en la vertiente cualitativa de la investigación las estrategias de generación de información son de dos tipos: (a) interactivas; (b) y las no interactivas. Dentro de las primeras se encuentran la observación participante, las entrevistas y las historias de vida. En las segundas están la revisión de documentos oficiales y personales. En el caso de la presente investigación predominó la observación participante como estrategia interactiva.

La investigación-acción es por definición participante. De ahí que la observación es forzosamente participante. Para ello se levantaron en diferentes momentos notas de campo en diarios y bitácoras (Martínez-Valerio, 2016), análisis, revisiones colegiadas de materiales didácticos y reconstrucción de procesos. 
De acuerdo con Macazaga y Rekalde (2005) y Rodríguez, Gil y García (1996), la observación en la que el investigador es también participante de los hechos que se estudian es un método de levantamiento de datos predominantemente interactivo, es decir, participativo. Lo anterior permite enterarse de opiniones, hechos y percepciones que no sería posible recabar utilizando otras técnicas. Dado que la metodología de investigación-acción es la que orientó de manera predominante el esfuerzo de intervención en la UACJ, la observación participativa fue la técnica pertinente para recabar datos de manera diacrónica a lo largo del periodo académico de otoño (agosto-diciembre) del 2018.

La doctora Isabel Guzmán y el doctor Rigoberto Marín (Guzmán y Marín, 2015; Guzmán, Marín e Inciarte, 2014; Marín y Guzmán, 2012) propusieron que el andamiaje pedagógico se utilizara como guía para ayudar a la reflexión, organización y observación de aquello que facilita al desarrollo de competencias y para estructurar las secuencias didácticas necesarias para realizar las tareas, proyectos y situaciones problema que en conjunto representan la innovación pedagógica:

Así, cada profesor tiene la libertad de documentar su diseño de las tareas/proyectos de aprendizaje de la forma que considere más adecuada. También es importante mencionar que este Andamio de Presentación a los estudiantes y su diseño instruccional contiene todos los elementos trabajados en la Planeación de la docencia descritos en el primer andamio (Guzmán y Marín, 2018, p. 4).

Los andamios elaborados por Guzmán y Marín permitieron instrumentar los tres vectores del modelo ReCREA: el pensamiento complejo, la investigación-acción participativa y la integración de las TIC al modelo de enseñanza. Lo que se propuso observar en la intervención para la innovación educativa se presenta en la Tabla 10. 
Tabla 10. Referentes para la observación de la innovación educativa del Proyecto ReCREA.

\begin{tabular}{|c|c|c|}
\hline $\begin{array}{c}\text { Elementos de } \\
\text { planeación de la } \\
\text { enseñanza del } \\
\text { Seminario ReCREA } \\
\text { (6 pasos) }\end{array}$ & $\begin{array}{c}\text { Tipología de los elementos } \\
\text { de cada secuencia didáctica } \\
\text { de la Guía para los } \\
\text { estudiantes } \\
\text { (Paso 6: presentación a los } \\
\text { estudiantes) }\end{array}$ & $\begin{array}{l}\text { Dispositivos y } \\
\text { elementos de la } \\
\text { Guía para los } \\
\text { estudiantes } \\
\text { (Paso 6: presentación } \\
\text { a los estudiantes) }\end{array}$ \\
\hline $\begin{array}{l}\text { La competencia } \\
\text { de salida y } \\
\text { competencias } \\
\text { constitutivas }\end{array}$ & $\begin{array}{l}\text { Las competencias que } \\
\text { se busca desarrollar o } \\
\text { contribuir a su desarrollo } \\
\text { con la asignatura }\end{array}$ & $\begin{array}{l}\text { I. Presentación y } \\
\text { descripción del } \\
\text { curso. (Competencia } \\
\text { docente de } \\
\text { transposición } \\
\text { didáctica) }\end{array}$ \\
\hline $\begin{array}{l}\text { Las tarea }(\mathrm{s}) / \\
\text { proyecto(s) } \\
\text { (específicos) }\end{array}$ & $\begin{array}{l}\text { A. Situaciones problema o } \\
\text { tarea/proyecto (contexto, } \\
\text { información y función) }\end{array}$ & \multirow{4}{*}{$\begin{array}{l}\text { II. Dispositivo de } \\
\text { formación } \\
\text { (Competencia } \\
\text { docente de diseño } \\
\text { de la docencia) }\end{array}$} \\
\hline $\begin{array}{l}\text { Los contenidos y } \\
\text { actividades del } \\
\text { curso }\end{array}$ & $\begin{array}{c}\text { B. Actividades de } \\
\text { aprendizaje (del curso) }\end{array}$ & \\
\hline $\begin{array}{l}\text { Los productos } \\
\text { esperados }\end{array}$ & $\begin{array}{l}\text { C. Evidencias de } \\
\text { desempeño }\end{array}$ & \\
\hline $\begin{array}{l}\text { La información } \\
\text { teórica, estratégica, } \\
\text { procedimental y las } \\
\text { prácticas requeridas } \\
\text { para la ejecución de } \\
\text { la tarea }\end{array}$ & D. Material de apoyo & \\
\hline $\begin{array}{l}\text { Los criterios de } \\
\text { evaluación }\end{array}$ & Los criterios de evaluación & \multirow{3}{*}{$\begin{array}{l}\text { III. Dispositivo de } \\
\text { evaluación } \\
\text { (Competencia de } \\
\text { evaluación del logro) }\end{array}$} \\
\hline $\begin{array}{l}\text { Los criterios de } \\
\text { calificación }\end{array}$ & Los criterios de calificación & \\
\hline $\begin{array}{l}\text { Los calendarios } \\
\text { de actividades } \\
\text { y entregas de } \\
\text { productos }\end{array}$ & $\begin{array}{l}\text { Los calendarios de } \\
\text { actividades y entregas de } \\
\text { productos }\end{array}$ & \\
\hline
\end{tabular}




\begin{tabular}{ccc}
$\begin{array}{c}\text { Bibliografía básica y } \\
\text { complementaria }\end{array}$ & $\begin{array}{c}\text { Referencias básicas y } \\
\text { complementarias }\end{array}$ & $\begin{array}{c}\text { Referencias } \\
\text { bibliográficas }\end{array}$ \\
\hline
\end{tabular}

Fuente: Guzmán, I. y Marín, R. (2018). 2. Andamio Guía de Estudiantes. Chihuahua, México: Universidad Autónoma de Chihuahua (material de trabajo no publicado).

Guzmán y Marín (2018) continúan explicando:

En la primera columna de la ... tabla [10] se describen los ocho elementos de un curso de conformidad con las actividades descritas en los cinco pasos de la Planeación de la Docencia mencionados en el primer andamio; en la segunda columna se propone una tipología para desarrollar secuencias didácticas cuya integración permite estructurar una guía académica para los estudiantes; finalmente, la tercera columna agrupa los elementos de cada secuencia en dos dispositivos: uno de formación y otro de evaluación, los cuales en conjunto tienden al desarrollo y evaluación de competencias académicas (Guzmán y Marín, 2018, p. 4-5).

Guzmán y Marín (2018) proponen la guía esquematizada que se presenta en la Figura 2 para la observación y evaluación del avance pedagógico con el grupo de estudiantes. 
Figura 2. Guía para instrumentar, observar y apreciar la innovación didáctica.

Elementos para la presentación de la tarea/situación compleja de aprendizaje

Caso/Tarea compleja/Situación/Proyecto

Desempeños (¿cómo se sabe que se resuelve correctamente y con calidad? Incluye la solución, argumentos, actitudes, procedimientos, etc.)

Selección de información con base en análisis de tipos de desempeño.

Evaluación de la resolución del caso / tarea compleja / proyecto / situación (matriz de valoración con los productos y evidencias de los objetivos de desempeño).

Actividades previas a la resolución del caso: por ejemplo lecturas, investigación bibliográfica, clase magistral, visitas, etc.

Fuente: Guzmán, I. y Marín, R. (2018). 2. Andamio Guía de Estudiantes. Chihuahua, México: Universidad Autónoma de Chihuahua (material de trabajo no publicado).

Entrevistas, encuesta y trabajos de los(as) estudiantes. Casanova (1998) plantea que:

En cualquier caso, las técnicas empleadas para la obtención de datos han de favorecer que ésta resulte todo lo rigurosa, sistemática, controlada y completa que se considere necesario en orden a que el modelo de evaluación planteado cumpla sus finalidades; es decir, para que 
permita mejorar el proceso de enseñanza y aprendizaje durante su puesta en práctica y para que los resultados que se obtengan por su medio [...] resulten lo suficientemente fiables, válidos, objetivos $y$, por lo tanto, útiles para el alumnado y el profesorado (p. 142).

Siguiendo la anterior línea de razonamiento, Casanova (1998) propone las siguientes técnicas para el levantamiento de datos: (a) la observación; (b) la entrevista; (c) la encuesta; (d) la sociometría; (e) el coloquio; (f) y los trabajos de los alumnos. Del listado anterior, las técnicas utilizadas para recabar datos del desarrollo de la innovación educativa fueron la observación, la entrevista, la encuesta, los trabajos de los estudiantes y adicionalmente el uso de los andamios pedagógicos. La primera técnica ya quedó explicada en el apartado anterior. En relación con los incisos "b" y "c" se aplicó una encuesta mediante entrevista a los estudiantes en relación al siguiente perfil socio-demográfico: ocupación actual, lugar de empleo, edad, localidad de nacimiento, estado civil, número de hijos y expectativas al egresar del programa de Licenciatura en Educación. A lo largo del periodo académico se tuvieron de manera recurrente entrevistas individuales con los estudiantes para monitorear sus avances en la experiencia de innovación educativa, clarificar dudas y valorar su comprensión de las temáticas abordadas.

En relación con el análisis de las evidencias de los trabajos de los estudiantes, se consideraron las tareas periódicas; los ejercicios y/o actividades de los estudiantes tanto áulicas como extra-áulicas; y se añadió el proyecto transversal que se realizó en todo el periodo académico. Dichas evidencias constituyeron "una fuente de datos insustituible para disponer de la información precisa en los procesos de evaluación” (Casanova, 1998, p. 155). Esa fuente de información fue de alto valor puesto que en el rol de profesor como investigador, este posee amplia experiencia para su apreciación.

Los trabajos que se utilizaron en la presente intervención para valorar los logros de aprendizaje por parte de los estudiantes, identificar las dificultades e interpretar las posibles causas tanto de los 
logros educativos como de las dificultades del aprendizaje que se presentaron se anotan en la Tabla 11.

Tabla 11. Trabajos académicos para el desarrollo y evaluación de las competencias de los estudiantes.

Entrega de reportes académicos semanales que incluyeron: (a) la síntesis de ideas principales de uno de los enfoques filosóficos vistos en clase durante la semana correspondiente; (b) la opinión personal fundamentada en donde se argumentaron las implicaciones del enfoque filosófico en su vida personal; (c) las implicaciones del enfoque filosófico en la práctica educativa en determinado nivel educativo a nivel de nuestra entidad federativa (Chihuahua) o de nuestro país (México).

Presentación oral y dinámica vivencial. Cada estudiante realizó la presentación oral de una perspectiva filosófica en clase. Se pidió que la presentación tuviera un componente vivencial que propiciara el aprendizaje significativo.

Utilización y evaluación del "andamio pedagógico" para implementar una práctica de innovación educativa consistente en el análisis de una política pública en educación en México aplicando una perspectiva filosófica.

Fuente: elaboración propia a partir del andamio pedagógico (Guzmán y Marín, 2018).

\section{APRECIACIÓN DE AVANCES DEL PROYECTO RECREA: 2018-AÑO 1}

La intención del Proyecto ReCREA a nivel nacional es que su horizonte de implementación abarque cuatro años. Los resultados en su primer año de implementación en la región noroeste se pueden estructurar bajo tres ópticas generales que se describen a continuación.

\section{MODELO PROPIO DEL PROYECTO RECREA}

Una forma de apreciar los logros de la presente intervención es basándose en el modelo presentado en la Figura 1 previamente donde se pueden evaluar los avances en cada uno de los tres segmentos principales que son: (1) el desarrollo del pensamiento complejo; (2) la modificación de los procesos sociales a través de la innovación e investigación-acción participativa; (c) y el uso, apli- 
cación e integración en extenso de las TIC en la vida académica tanto del profesorado como de los(as) estudiantes.

\section{PLANEACIÓN OPERATIVA DEL GRUPO DE TRABAJO DE LA REGIÓN NOROESTE}

La segunda opción para valorar los avances del presente proyecto es revisando los compromisos y metas establecidos por la red regional. El colectivo de investigadores de las dos UPE y de las tres escuelas normales de Chihuahua y Sonora desarrollamos una lista de compromisos para la implementación del proyecto de investigación Sistematización de experiencias educativas en comunidades de práctica innovadoras. Para ello se visualizaron las siguientes fases: (a) la construcción de la propuesta de experiencias educativas innovadoras, (b) la sistematización de experiencias educativas (ciclo 1), (c) el diseño de la docencia para una práctica educativa innovadora, (d) el proceso de intervención a través de los Pro-Facilitadores (PF) y de los Facilitadores Regionales (FR), (e) la sistematización de las experiencias educativas (ciclo 2), (f) y la intervención en el aula. En esta segunda opción se tendrían que valorar los adelantos para cada una de las etapas listadas aquí y concertar a todos los integrantes del colectivo regional en esta tarea.

\section{MODELO CÍCLICO DE INVESTIGACIÓN-ACCIÓN (I-A) DE 4 FASES}

La tercera opción de presentación de los resultados es utilizando las cuatro fases propuestas por Kemmis y McTaggart (1992, 2007) para realizar investigación para la transformación en educación aplicando la metodología de la investigación-acción participativa (innovación-docencia). Dichas etapas son las siguientes: (a) la planeación de una acción o series de acciones concretas, (b) la implementación de la acción o de las series de acciones acordadas, (c) la observación sistemática de la conducción de las acciones y de sus diversos impactos, (d) y la reflexión 
y socialización crítica, con base en lo observado, con la finalidad de planear una acción o secuencia de acciones nuevas.

Para el presente reporte se utilizará, por razones de economía en la exposición, la tercera opción, la del Modelo Cíclico de I-A de 4 fases para presentar los resultados del primer año de desarrollo del Proyecto ReCREA con la participación de dos académicos de la Universidad Autónoma de Ciudad Juárez.

Los resultados se presentan a continuación considerando los siguientes tres niveles de desagregación:

- A nivel regional con el grupo de Pro-Facilitadores y de Facilitadores Regionales.

- A nivel de los investigadores adscritos a través de la UACJ al CA-54 Estudios de Educación y Ciencias Sociales.

- Y finalmente a nivel áulico de la clase de Filosofía de la Educación (FE).

La forma de presentación previa se esquematiza en la Tabla 12 a continuación.

Tabla 12. Estructura para la presentación de los avances del Proyecto ReCREA.

\begin{tabular}{|c|c|c|c|}
\hline & \multicolumn{3}{|c|}{ Desagregación por amplitud de la investigación } \\
\hline & Colectivo regional & $\begin{array}{l}\text { Grupo de in- } \\
\text { lvestigación en } \\
\text { la universidad }\end{array}$ & Áulico \\
\hline Planeación & \multirow{4}{*}{$\begin{array}{c}\text { Avances a nivel } \\
\text { - del colectivo re- } \\
\text { gional }\end{array}$} & \multirow{4}{*}{$\begin{array}{c}\text { Avances } \\
\text { a nivel del } \\
\text { equipo de } \\
\text { investigación } \\
\text { del Proyecto } \\
\text { ReCREA de la } \\
\text { Universidad } \\
\text { Autónoma de } \\
\text { Ciudad Juárez }\end{array}$} & \multirow{4}{*}{$\begin{array}{l}\text { Avances a nivel } \\
\text { áulico en la impar- } \\
\text { tición de la clase } \\
\text { de Filosofía de la } \\
\text { Educación del De- } \\
\text { partamento de Hu- } \\
\text { manidades (ICSA) }\end{array}$} \\
\hline Implementación & & & \\
\hline $\begin{array}{c}\text { Observación siste- } \\
\text { mática }\end{array}$ & & & \\
\hline $\begin{array}{l}\text { Reflexión y sociali- } \\
\text { zación crítica }\end{array}$ & & & \\
\hline
\end{tabular}

Fuente: elaboración propia. 
Apreciación de avances a nivel del colectivo regional del proyecto ReCREA-RNO. Se formó un colectivo integrado por dos UPE y por tres escuelas normales de los estados de Chihuahua y Sonora. Participamos académicos de cinco instituciones de educación superior y se tuvieron seis reuniones bimestrales durante el año 2018. Se completaron de la anterior manera seis ciclos generales de investigación-acción donde se diseñaron dos andamios pedagógicos para instrumentar los tres enfoques del Proyecto ReCREA: (a) el del pensamiento complejo; (b) el enfoque de investigación participativa en la enseñanza; (c) y finalmente el uso de las TIC. Dichos andamios fueron piloteados durante el semestre de primavera de 2018; la innovación educativa fue implementada de forma completa en el semestre de otoño de 2018 en las clases elegidas para dicho propósito en las instituciones participantes.

Todos los integrantes del colectivo regional tuvimos acceso al portal creado por la DGESU-SES (http: //cursos.acet-latinoamerica. org/recrea/). Ahí los participantes subimos como evidencias el andamio del estudiante y una propuesta inicial de evaluación, así como un informe parcial de actividades (Almeida, 2018) realizadas del Proyecto ReCREA-RNO en su primer año de implementación.

El conjunto de académicos recibimos la certificación como Facilitadores Regionales del Proyecto ReCREA, con la cual acudimos a diversas escuelas normales y centros de posgrado en educación de los estados de Chihuahua, Sonora, Sinaloa, Baja California y Baja California Sur para ampliar el alcance del proyecto. Se establecieron cartas de intención y una serie de acuerdos con los grupos de docentes y autoridades de las instituciones visitadas a las que se les impartió la capacitación para continuar con la implementación de la innovación educativa en el año 2 subsecuente correspondiente al 2019.

Apreciación de avances a nivel del equipo investigación del Proyecto ReCREA-RNO. Con el grupo de interés para la investigación adscrito al CA-54-UACJ se logró incrementar la colegiación entre dos integrantes del mismo. Se participó de manera conjunta en las reuniones regionales para recibir la capacitación como Facilitadores Regionales. Logramos dicha certificación expedida por la 
Dirección General de Educación Superior Universitaria. Iniciamos la interlocución con universidades públicas estatales y con escuelas normales de Baja California y Baja California Sur para difundir la metodología del Proyecto ReCREA. En diciembre de 2018 asistimos a Tijuana a la Escuela Normal Fronteriza Tijuana, y acudimos a La Paz, Baja California a la Benemérita Escuela Normal Urbana "Profr. Domingo Carballo Félix" para impartir la capacitación a los cuerpos académicos de dichas instituciones para que iniciaran la implementación en los periodos académicos subsecuentes. Ambos académicos hemos asumido una postura crítica en relación al proyecto y hemos realizado la adaptación del mismo a clases elegidas con dicho propósito en el Instituto de Ciencias Sociales y Administración (UACJ).

Los profesores involucrados hemos logrado mayor cercanía académica y un clima de confianza para retroalimentar de manera crítica nuestras actividades académicas. Lo anterior ha propiciado tanto la innovación educativa como la actualización en enfoques, contenidos y herramientas de investigación para la mejora de la educación superior en nuestra institución.

Apreciación de avances a nivel áulico en la clase de Filosofía de la Educación del Proyecto ReCREA-RNO. A nivel áulico, en la clase de FE se implementó el andamio para el estudiante con fines de investigación educativa. Se propuso como experiencia de innovación educativa que cada estudiante eligiera una política de educación pública en México para ser abordada de manera crítica desde una o varias posturas filosóficas. Se propuso que el cuestionamiento de la política se hiciera desde una o dos perspectivas filosóficas vistas en clase y considerando un entorno de complejidad y ambigüedad. Se pidió al grupo que utilizara en todo momento dispositivos electrónicos, accesos a bases de datos, bibliotecas virtuales y se estableció como medio de comunicación alterna los propios correos electrónicos del profesor y de los estudiantes (aplicación de las TIC).

Las y los estudiantes participaron también asumiendo el rol de investigadores de sus propios procesos. Revisaron el andamiaje, los materiales y los planteamientos académicos. Como con- 
secuencia de las revisiones realizadas por ellos(as) se hicieron modificaciones y adaptaciones a la propuesta de trabajo original presentada por el profesor, a los criterios de evaluación y al andamio pedagógico presentado al estudiante.

Los avances y dificultades que se presentaron derivados del presente proyecto de innovación educativa se describen en la Tabla 13.

Tabla 13. Información de valoración de la innovación educativa obtenida con diversas técnicas.

Tipología de los elementos Avances y dificultades que se identificaron de cada secuencia didáctica resultado del uso del andamio, observación, de la Guía para los estuentrevistas y bitácoras. diantes

(Paso 6: presentación a los estudiantes)

Las competencias que se busca desarrollar o contribuir a su desarrollo con la asignatura
El programa se centró sobre el desarrollo de una sola competencia. Se abandonó el esquema de que en un solo curso es factible y deseable el desarrollo de múltiples competencias. 
Los criterios de evaluación Las especificaciones muy concretas utilizaLos criterios de calificación das en el andamio del estudiante ayudaron $\overline{\text { Los calendarios de activida- }}$ a los estudiantes a clarificar las expectativas des y entregas de productos de estudio y de logros de aprendizaje. Lo anterior ayudó a que el $75 \%$ del grupo realizara satisfactoriamente las tareas, actividades y proyecto del curso.

La dosificación y la temporalización provistas a través del andamio del estudiante se consideraron adecuadas. El perfil del grupo de estudiantes fue en un rango de edad entre 19 y 28 años, $75 \%$ del grupo con empleo formal y con responsabilidad familiar baja. El perfil anterior, en una zona urbana fronteriza como lo es Ciudad Juárez y añadiendo una carga académica en exceso, provocó que los estudiantes contaran con poco tiempo para realizar las tareas académicas.

Lo anterior fue en detrimento del desarrollo de la competencia central diseñada para esta clase.

Referencias básicas y com- Las fuentes documentales se ampliaron en plementarias exceso. Los estudiantes reportaron no tener tiempo para hacer la lectura de todas las referencias documentales. Solicitaron limitarlas o dosificarlas de acuerdo con los intereses y proyectos de cada estudiante.

Fuente: Adaptación propia del original con la autorización de Guzmán, I. y Marín, R. (2018).

Por observación y lectura de los reportes semanales se pudo validar que los estudiantes lograron incrementar la capacidad para comprender y conceptualizar situaciones complejas. También se identificó una relación directamente proporcional entre el número de revisiones de un reporte académico y el mejoramiento de la calidad y del contenido del mismo. A mayor número de revisiones de los escritos de los estudiantes mejoraron sensiblemente la calidad de los mismos. Se infiere también que las competencias intelectuales asociadas con dicho desempeño han tenido mayor desarrollo. Por ello, se estima que el andamiaje diseñado para el Proyecto ReCREA estimula el desarrollo de las siguientes compe- 
tencias: (a) la definición de problemáticas, (b) y la vinculación del curso con otras áreas disciplinarias. Emergió la necesidad del trabajo conjunto y personalizado del profesor titular de la asignatura con cada uno de los estudiantes.

Los resultados cuantitativos finales de la intervención son los siguientes: dos estudiantes tuvieron que dejar la clase por razones personales; únicamente un estudiante no entregó el proyecto semestral. Doce estudiantes entregaron el reporte final; de ese total, únicamente una estudiante no tuvo una evaluación satisfactoria en su reporte. Con todos hubo una interacción a nivel de tutoría durante el periodo académico para conducirlos en la aplicación del andamio pedagógico.

\section{DELIBERACIÓN FINAL}

Limitaciones de la implementación del Proyecto ReCREA

La propuesta teórica y metodológica del Proyecto ReCREA a nivel nacional y a nivel estatal, en el estado de Chihuahua ha enfrentado las limitaciones que se explican a continuación.

- Se ha propuesto y se ha intentado aplicar simultáneamente una diversidad de enfoques metodológicos, los cuales, cada uno de ellos, justificarían un proyecto de investigación por sí solo, con la cobertura y con la temporalidad (4 años) que se le ha pretendido dar al actual trabajo. Algunos de los enfoques metodológicos que se ha buscado hacer converger simultáneamente son los siguientes: (a) la sistematización de experiencias educativas, (b) la formación de comunidades de práctica innovadora, (c) la investigación educativa para la innovación pedagógica, (d) y el modelo para el desarrollo y evaluación de competencias académicas. Dicha multiplicidad de enfoques de trabajo investigativo ha resultado en cierto grado de confusión con algunos académicos, con algunos colectivos e instituciones. Ello ha provocado lentitud en los tiempos de capacitación, difusión e implementación del Proyecto ReCREA. Asimismo, al 
interior de la Universidad Autónoma de Ciudad Juárez ha imposibilitado su mayor propagación en la comunidad universitaria por lo amplio de las metodologías y la pretensión de conjugarlas simultáneamente para buscar el propósito, teóricamente hablando, de mejorar la docencia en educación superior.

- En forma paralela y complementaria a la anterior crítica, el Proyecto ReCREA ha buscado la transformación de la práctica docente centrándose en los siguientes ejes que se plantearon teóricamente como "transversales": (a) un enfoque epistemológico sustentado en la perspectiva de la complejidad y en el desarrollo de competencias profesionales, (b) la incorporación de los avances de la investigación en el proceso y contenido de la enseñanza-aprendizaje, (c) el desarrollo de las competencias digitales, (d) la intervención e investigación en el aula, (e) y la sistematización de experiencias educativas (nuevamente). De forma reiterativa se ha hecho un planteamiento con múltiples enfoques tanto teóricos, metodológicos como pragmáticos. En los tiempos de la capacitación y divulgación del Proyecto ReCREA se ha presentado cierta confusión entre el profesorado, los grupos de investigación y las propias instituciones. Se presentan dudas en relación a cómo se puede implementar tanta variedad de enfoques en la práctica docente cotidiana en las diferentes disciplinas de enseñanza tanto universitaria como normal.

- Por otro lado, ha habido confusión en relación con el manejo del concepto de transversalidad. La transversalidad es un concepto que generalmente se aplica a nivel del plan de estudios, ya sea para su diseño o para el rediseño curricular. En contraste en el Proyecto ReCREA, se le ha utilizado, en ciertos momentos, como una característica del ejercicio docente a nivel individual, lo que parece entrar en contradicción con el manejo académico general del tecnicismo citado. 
- El Proyecto ReCREA ha tenido como una de sus estrategias principales el colocar en interacción a los docentes de educación superior de escuelas normales y de universidades públicas estatales. Efectivamente, se ha convocado a académicos de ambos subsistemas de educación superior. Lo anterior ha provocado cierto desorden en relación a qué subsistema guiará los trabajos. La experiencia docente de académicos de ambos subsistemas ha resultado ser igualmente valiosa y legítima. Sin embargo, en los grupos de trabajo ha habido ambivalencia en relación a qué directrices se deben seguir, si las marcadas por el subsistema de escuelas normales, o las indicadas por el subsistema de universidades públicas estatales. Lo anterior ha limitado la implementación del Proyecto ReCREA en algunos grupos e instituciones.

- La vertiente filosófica del pensamiento complejo en el Proyecto ReCREA se ha encontrado sobreenfatizada. Si bien el pensamiento de Morin es relevante como postura crítica de la enseñanza universitaria y normal parcelaria y burocrática, dicho énfasis ha limitado en cierto grado los procesos de reflexión, investigación y deliberación del Proyecto ReCREA. También ha prevenido la inclusión de otras perspectivas teoréticas de las que somos portadores participantes del Proyecto ReCREA y que ha faltado tiempo para su revisión e inclusión como parte de los referentes teóricos del proyecto.

- En el Proyecto ReCREA se han visto de manera predominante académicos de las áreas de humanidades, ciencias sociales, educación, artes y educación normal. Ha faltado incluir en la conducción global del proyecto a profesionales que se desempeñan también como maestros de otros sectores y disciplinas como por ejemplo las siguientes: (a) agronomía, veterinaria y afines; (b) bellas artes; (c) ciencias de la salud; (d) economía, administración, contaduría y afines; (e) ingeniería, arquitectura, urbanismo y afines; y (f) matemáticas y ciencia naturales. 


\section{RECOMENDACIONES PARA INVESTIGACIÓN FUTURA Y PARA LA CONTINUACIÓN DEL PROYECTO}

Con base en la experiencia derivada de la implementación del Proyecto ReCREA y a la revisión de su propuesta teórica-metodológica, es conveniente expresar las siguientes recomendaciones para proyectos que tengan una envergadura similar en México o en el estado de Chihuahua. Estas recomendaciones también pueden ser asumidas por los integrantes de la actual Red CREA.

- Hacer una invitación que abarque al resto del universo de subsistemas de educación superior que operan en el estado de Chihuahua y en la República Mexicana. Para ello se sugiere que en esta red se invite también a grupos de investigación y académicos de los siguientes subsistemas: universidades federales, universidades públicas estatales (ya incluido), universidades públicas estatales con apoyo solidario, universidades tecnológicas, universidades politécnicas, universidades interculturales, institutos tecnológicos federales y descentralizados, escuelas normales públicas (ya incluido), escuelas normales privadas, centros de investigación Conacyt, e instituciones de educación superior particulares.

- Internacionalizar la Red CREA iniciando la invitación a instituciones de educación superior de otros países con los que México tiene mayor intercambio comercial y económico.

- Realizar una propuesta teórica-metodológica reduciendo la cantidad de ejes y abordajes metodológicos que se aspira implementar simultáneamente. Esta recomendación permitirá: (a) que los esfuerzos de capacitación a los cuerpos de investigación y docencia sea más eficaz, (b) que las expectativas sean más comunicables, (c) y a que su implementación se dé con mayor fluidez.

- Independizar la red del sector gubernamental. Buscar fuentes de financiamiento alternativas para apoyar el desarrollo 
de las agendas de trabajo que permitan continuar con el trabajo académico y de investigación. Realizar una gestión para la obtención de recursos desde las comunidades académicas para las mismas comunidades académicas.

- Dar la bienvenida a perspectivas epistémicas diferentes. No sobreenfatizar en demasía el pensamiento complejo moriniano.

- Ampliar las redes de colaboración para propiciar el intercambio permanente de proyectos de innovación didáctica en educación superior de cualquier subsistema de México y del mundo.

- Generar de manera permanente redes y grupos de colaboración que favorezcan el intercambio en el mediano y largo plazo de métodos de investigación, epistemologías, estrategias educativas, técnicas y recursos didácticos que propicien la mejora de la calidad y de la innovación en educación superior.

- Ampliar la población meta a profesionistas de otros sectores y discplinas: (a) agronomía, veterinaria y afines; (b) bellas artes; (c) ciencias de la salud; (d) economía, administración, contaduría y afines; (e) ingeniería, arquitectura, urbanismo y afines; y (f) matemáticas y ciencia naturales.

\section{CONCLUSIÓN}

El modelo de innovación educativa propuesto por la Subsecretaría de Educación Superior (y coordinado por la DGESPE y la DGESU, 2017) puede ser útil para mejorar la práctica docente en educación tanto en el subsistema de las UPE como en el subsistema de las escuelas normales. Lo anterior es viable al introducir referentes de innovación pedagógica como lo son:

- El uso del andamio para la planeación por parte del docente

- El uso de lecturas complementarias representativas de diferentes perspectivas 
- La investigación documental en motores avanzados de búsqueda digitales

- Las presentaciones orales por parte de los estudiantes complementados con dinámicas vivenciales y diseñados en conjunto con el profesor titular de la materia

- La integración de los propios estudiantes a los grupos de investigación de los docentes universitarios y normalistas

- La revisión periódica de los reportes semanales, la retroalimentación personalizada y constante

- El mayor acercamiento académico del docente con sus estudiantes en clase

- Una revisión más intensa y meticulosa del trabajo académico de los estudiantes por parte del docente

- La focalización en las cátedras al desarrollo de competencias específicas ofreciendo el suficiente tiempo y atención para su ejercicio

- Y el uso del andamio pedagógico asumido por el estudiante de manera crítica

Con el Proyecto ReCREA se ha logrado crear un ambiente de colegiación académica en las siguientes esferas: (a) intrainstitucional; (b) interinstitucional, entre cuerpos académicos de distintas universidades; (c) intersectorial, entre cuerpos académicos de escuelas normales y UPE; (d) interestatal, mediante reuniones con cuerpos académicos e investigadores de otras entidades federativas de la región NO; (e) y nacional, propiciando la colegiación con los grupos de investigación del resto del país: noreste, suroeste, sureste y occidente. Estas actividades de intercambio académico han enriquecido y ampliado las visiones educativas, las posturas y estrategias didácticas, así como los métodos de investigación educativa de manera amplia para todos los(as) participantes del Proyecto ReCREA.

La interacción entre docentes investigadores tanto del sector universitario como del de escuelas normales es crucial para el desarrollo de la innovación pedagógica, ya que ocurre un aprendizaje interinstitucional y simultáneamente interdisciplinario. Este 
acercamiento entre ambos subsistemas es histórico, ya que tradicionalmente se habían mantenido distanciados. También ha resultado que dicho acercamiento es tomado de manera receptiva por los distintos grupos de investigadores y que las posibilidades de interacción e intercambio académico son múltiples.

Asimismo, la utilización de otras formas de retroalimentación no asociadas de manera tradicional con la enseñanza (el hincapié en la redacción, en la aplicación de actividades vivenciales y en la adquisición de competencias profesionales), en el caso de la clase de $\mathrm{FE}$, incrementa la capacidad del estudiante para comprender y conceptualizar situaciones complejas, estimula el desarrollo de las competencias de definición de problemáticas y vinculación del curso con otras áreas disciplinarias. Lo anterior ayuda al estudiante a desarrollar competencias de diagnóstico y propuesta educativa que le permitirán un mejor desempeño en los centros de trabajo del sector educativo en el futuro. Es decir, desarrolla su capacidad de aprendizaje para situaciones nuevas en ambientes inciertos y ambigüos.

Es importante que el modelo propuesto por la Subsecretaría de Educación Superior (DGESPE-DGESU) continúe generalizándose de manera crítica, reflexiva, flexible y dinámica en diversas asignaturas en un mayor número de universidades, de escuelas normales e incluso de instituciones de educación superior de otros subsistemas de México y de otros países. Si bien el modelo inicial con tres aristas (el pensamiento complejo, la investigación-acción y el uso de las TICs) se recomienda que sea simplificado, puede ser utilizado como propuesta inicial del trabajo académico sujeto a revisión. Lo anterior permitirá ir modificando y adaptando los planteamientos conceptuales y las herramientas didácticas propuestas, para que sea viable aplicarlos en contextos distintos, por las comunidades académicas de diferentes sectores de la educación superior tanto de México como de otros países. 


\section{REFERENCIAS}

Almeida U., R. (2018). Informe del primer ciclo del Proyecto RECREA-RNO del Cuerpo Académico "Educación y Ciencias Sociales" (UACJ-CA-54). Ciudad Juárez, Chihuahua: informe parcial sin publicar.

Almeida U., R., Arias, E., Curiel, C., Díaz, P., Flores, E. ł, Muttio, C. y Priego, A. F. (revisado por Almeida \& Labrado) (2009). Manual de presentación de trabajos y exámenes, Ciudad Juárez, Chihuahua: manual sin publicar.

Aseguramiento de la Calidad en la Educación y en el Trabajo, S. C. (2010a). Diseño de tareas y proyectos, México: Proyecto Aula/Universidad Veracruzana.

Aseguramiento de la Calidad en la Educación y en el Trabajo, S. C. (2010b). Pasos para el diseño de tareas/proyectos de aprendizaje para el desarrollo de competencias y pensamiento complejo, México: Proyecto Aula/Universidad Veracruzana.

Aseguramiento de la Calidad en la Educación y en el Trabajo S. C. (2010c). Diez pasos para el aprendizaje complejo: Un acercamiento sistemático al diseño instruccional de los cuatro componentes. En Jeroen J. G., van Merriënboer y Paul A. Kirschner. Ten Steps to Complex Learning: A Systematic Approach to Four-Component Instructional Design. (2007). Nueva York: Lawrence Erlbaum Associates, Inc., Publishers.

Bausela H., E. (2004). La docencia a través de la investigaciónacción. Recuperado de: https: //rieoei.org.

Bolívar, A., de la Cruz, J. M. y Domingo, J. (2001). La investigación biográfico-narrativa en educación: enfoque y metodología, Madrid: La Muralla.

Casanova, M. A. (1998). La evaluación educativa, España: Editorial Muralla.

Cisneros-Cohemour, E. J., García-Cabrero, B., Luna S., E. y Marín U., R. (eds.). (2012). Evaluación de competencias docentes en la educación superior, México: Red para el Desarrollo y Evaluación de Competencias Académicas/Juan Pablos Editor. 
Cruz L., Y. y Cruz L. A. K. (2008). La educación superior en México tendencias y desafíos. Revista de Avialaçäo de Educación Superior, 13(2), 293-311.

Dirección General de Educación Superior (2018). Comunidades de práctica para la innovación en la docencia en educación superior, México: Subsecretaría de Educación Superior/Secretaría de Educación Pública. Recuperado de: http://cursos.acet-latinoamerica.org/recrea/

Duarte J. R. (2017). Universidad Autónoma de Ciudad Juárez. Quinto informe de actividades 2016-2017, Ciudad Juárez, Chihuahua: Universidad Autónoma de Ciudad Juárez.

Elliot, J. (1993). El cambio educativo desde la investigación acción, Madrid: Morata.

Giroux, S. y Tremblay, G. (2004). Metodología de las ciencias humanas. La investigación en acción. Trad. Beatriz Álvarez Klein, México: FCE.

Gulikers, J.T.M., Bastiaens, T.J. y Kirschner, P.A. (2004). A FiveDimensional Framework for Authentic Assessment. Educational Technology Research and Development, 52(3), 67-86.

Guzmán, I. y Marín, R. (2018). 2. Andamio Guía de Estudiantes, Chihuahua, México: Universidad Autónoma de Chihuahua (material de trabajo no publicado).

Guzmán, I. y Marín, R. (2015). Competencias y formación universitaria: dos experiencias de intervención en el aula. En Marín, R., Guzmán, I., Inciarte, A. y Araya, E. (eds.), Intervenir e investigar en el aula: Experiencias en la formación de Profesores (20-63), Argentina: Alfagama y REDECA.

Guzmán, I., Marín, R. e Inciarte, A. (2014). Innovar para transformar la docencia universitaria. Un modelo para la formación de competencias. (S. Ediciones Astro Data, Ed.) (Primera edición). Jenkins, A., Healey, M. y Zetter, R. (2007). Linking teaching and research in disciplines and departments, York, Reino Unido: The Higher Education Academy.

Kemmis, S. (1998). El currículum más allá de la teoría de la reproducción, Madrid: Morata. 
Kemmis, S. y McTaggart, R. (1992). Cómo planificar la investigación acción, Barcelona: Laertes.

. (2007). Participatory Action Research: Communicative Action and the Public Sphere. En Denzin, N. y Lincoln, Y. (eds). Strategies of Qualitative Inquiry (271-330), Thousand Oaks: Sage.

Kirschner, P.A., Sweller, J. y Clark, R. E. (2006). Why Minimal Guidance During Instruction Does Not Work: An Analysis of the Failure of Constructivist, Discovery, Problem-Based, Experiential, and Inquiry-Based Teaching. Educational Psychologist, 41(2), 75-86.

Latorre, A. (2005). La investigación-acción. Conocer y cambiar la práctica educativa, Barcelona: Graó.

Macazaga, A. y Rekalde, I. (2005). El proceso de formación a través de la investigación participativa: una experiencia en torno al deporte escolar. Guiniguada, (14), 141-156.

Marín, R. y Guzmán, I. (2012). Formación-evaluación: una propuesta para el desarrollo y evaluación de competencias docentes. En Cisneros Chacón, E., García Cabrero, B., Luna, E. y Marín Uribe, R. (coords). Evaluación de competencias docentes en la educación superior, México: Juan Pablos editor.

Martínez-Valerio. L. (2016). La bitácora como forma de documentación de la actividad en redes sociales online. Documentación de las Ciencias de la Información, (39), 165-175. Recuperado el 18 de Noviembre de 2018 de: http://dx.doi.org/10.5209/ DCIN.54415

Morin, E. (1994). Introducción al pensamiento complejo, Barcelona: Gedisa.

Morin, E., Roger C., E. y Domingo M., R. (2002). Educar en la era planetaria. El pensamiento complejo como método de aprendizaje en el error y la incertidumbre humana. Valladolid: Secretariado de Publicaciones e Intercambio Editorial/Universidad de Valladolid.

Nicolescu, B. (1996). La transdiciplinariedad, París: Ediciones Du Rocher.

Nicolescu, B. (2006). Transdisciplinariedad: pasado, presente y futuro. Primera parte. Visión Docente con-ciencia, (31), 15-31. 
---- (2006). Transdisciplinariedad: pasado, presente y futuro. Segunda parte. Visión Docente con-ciencia, (32), 14-33.

Norton, L. S. (2009). Action research in Teaching \& Learning: a practical guide to conducting pedagogical research in universities, Londres: Routledge.

Osorio, S. N. (2012). El pensamiento complejo y la transdisciplinariedad: fenómenos emergentes de una nueva racionalidad. Investigación y Reflexión, 20(1), 269-291.

Padilla C. M. T. y Gil F. J. (2008). La evaluación orientada al aprendizaje en la Educación Superior: condiciones y estrategias para su aplicación en la docencia universitaria. Revista española de pedagogía, LXVI (241), 467-486.

Rekalde, I., Vizcarra, M. T. y Macazaga, A. M. (2014). La observación como estrategia de investigación para construir contextos de aprendizaje y fomentar procesos participativos. Educación, XXI, 17(1), 199-220.

Rodríguez, G., Gil, J. y García, E. (1996). Metodología de la investigación cualitativa, Málaga: Aljibe.

Rodríguez G., D. y Valldeoriola R., J. (2009). Metodología de la investigación, Barcelona: Fundación para la Universitat Oberta de Catalunya.

Roegiers, X. (2010). Pedagogía de la integración. Competencias e integración de los conocimientos en la enseñanza, México: FCE.

Shu, X. (2016) An Action Research on TPACK's Influence on Teachers of National Open University: Exemplified with an English Teacher of Zhejiang Radio and TV University. Open Access Library Journal, 3, 1-6.

Sluijsmans, D. M. A., Straetmans, G. J. J. M. y Merriënboer, J. J. G. van. (2008). Integrating authentic assessment with competence-based learning in vocational education: the Protocol Portfolio Scoring. Journal of Vocational Education and Training, 60 (2), 159-172.

The Boyer Commission for Educating Undergraduates in the Research University. (1998). Reinventing Undergraduate Educa- 
tion: A Blueprint for America's Research University, Princeton, NJ: Carnegie Foundation for the Advancement of Teaching.

Universidad Autónoma de Ciudad Juárez. (2019). Quiénes somos. Estructura académica. Humanidades. Licenciatura en educación. Perfil de egreso, Ciudad Juárez, Chihuahua: Universidad Autónoma de Ciudad Juárez. Recuperado de http: //www.uacj.mx/ICSA/Humanidades/LE/Paginas/default. aspx.

Van Merriënboer, J. J. G. y Kirschner, P. A. (2010). Diez pasos para el aprendizaje complejo: un acercamiento sistemático al diseño instruccional de los cuatro componentes (Traducción al español autorizada a Aseguramiento de la Calidad en la Educación y en el Trabajo, S.C. por Lawrence Erlbaum Associates, Inc., Publishers), Universidad Veracruzana: Aseguramiento de la Calidad en la Educación y en el Trabajo, S. C.

Wikimedia Foundation, Inc. Ciudad Juárez. Recuperado de https: //en.wikipedia.org/wiki/Ciudad_Ju\%C3\%A1rez. 